\title{
Simple and High-sensitivity Colorimetric Analysis of Cadmium using Homogeneous Liquid-Liquid Extraction
}

Atsushi MANAKA, ${ }^{* \dagger}$ Yumemi UENO, ${ }^{*}$ Masamoto TAFU, ${ }^{*}$ and Takeshi $\mathrm{KATO}^{* *}$

* Department of Applied Chemistry and Chemical Engineering, National Institute of Technology, Toyama College, 13 Hongo, Toyama 939-8630, Japan

** National Institute for Materials Science, 1-2-1 Sengen, Tsukuba, Ibaraki 305-0047, Japan

$\dagger$ National Institute of Technology, Toyama College, 13 Hongo, Toyama 939-8630, Japan

E-mail: manaka@nc-toyama.ac.jp 


\begin{abstract}
Colorimetric determination with dithizone (diphenylthiocarbazone) is a simple method that has been applied to analyze cadmium $(\mathrm{Cd})$ ions in water. However, determining concentrations close to the environmental standard value $\left(3 \mu \mathrm{gL}^{-1}\right)$ using this method is difficult because of its low sensitivity. Herein, we exploited the phase separation phenomenon to generate a small amount of the extraction phase by adding a mixture of 2-propanol and a small amount of chloroform to a water sample, as a pre-concentration method for the $\mathrm{Cd}(\mathrm{II})$ ions. The obtained $\mathrm{Cd}(\mathrm{II})$-dithizone complex was successfully extracted into this phase. We then constructed a simple and highly sensitive colorimetric analysis method for the ppb level of $\mathrm{Cd}(\mathrm{II})$ using this phase separation technique.
\end{abstract}




\section{Introduction}

Cadmium ions $(\mathrm{Cd}(\mathrm{II}))$ are essential in several industrial applications, such as alloys, pigments, batteries, and reactor control rods ${ }^{1}$. However, water pollution caused by $\mathrm{Cd}(\mathrm{II})$ is a severe environmental issue in some regions of the world ${ }^{2-4}$ because $\mathrm{Cd}(\mathrm{II})$ causes serious health problems such as Itai-Itai disease. Therefore, the World Health Organization (WHO) and Japanese environmental standards define the standard value of $\mathrm{Cd}(\mathrm{II})$ concentration in water environments to be $3.0 \mu \mathrm{gL}^{-1}$ or less, and monitoring the concentration of cadmium in water resources was made mandatory by $\mathrm{WHO}^{5}$.

Instrumental analyses, such as inductively coupled plasma optical emission spectrometry (ICP-OES) are frequently used analytical methods for the determination of Cd(II) having a high level of sensitivity ${ }^{6}$. However, these techniques require expensive instruments and involve complex operations. On the contrary, colorimetric determination with dithizone has been widely used as a simple analytical method for the determination of $\mathrm{Cd}(\mathrm{II})^{7,8,9}$. This method exploits a color reaction (colorless to red) to quantify the Cd (II) concentration of sample solutions under basic conditions. However, determining $\mathrm{Cd}(\mathrm{II})$ concentrations close to the environmental standard value $\left(3.0 \mu \mathrm{gL}^{-1}\right)$ via this method is difficult because of its low sensitivity.

To improve the sensitivity of colorimetric analysis with dithizone, we herein focused on the phase separation phenomenon. Specifically, we explored homogeneous liquid-liquid extraction, whereby a small amount of the extraction phase is generated $^{10-12}$. Through homogeneous liquid-liquid extraction, the analyte can be concentrated from a milliliter-scale sample to a microliter-scale extraction phase in a short period of time. This fact indicates that this method is a simple preconcentration method yielding a high concentration rate. Therefore, this method has been applied to increase the sensitivity of several instrumental analysis techniques ${ }^{10-14)}$ such as gas 
chromatography-mass spectrometry $(\mathrm{GC}-\mathrm{MS})^{1 \theta}=$. Recently, it was found that a mixture of water, 2-propanol, and chloroform generates a similar microscale water-insoluble extraction phase, and a Cd(II)-dithizone complex can be concentrated. Therefore, the purpose of this study was to develop a highly sensitive colorimetric analysis technique for $\mathrm{Cd}(\mathrm{II})$ using phase separation with water, 2-propanol, and chloroform, and to evaluate the proposed method for environmental analysis.

\section{Experimental}

\section{Reagents and Apparatus}

Standard Cd(II) solution, Dithizone, chloroform, and 2-propanol, and Sodium carbonate was obtained from Fuji Film Wako Pure Chemical Industries Ltd., Japan Standard river water was obtained from the National Institute of Advanced Industrial Science and Technology (AIST); this standard contained $1.0 \mathrm{ppb}$ of Cd. An AV-630 spectrophotometer (JASCO Corp.) was used to measure the absorbance. An MCF-1350 centrifugal separator (LMS, Co., Ltd.) was used to promote phase separation.

\section{Procedure}

First, $2 \mathrm{~mL}$ of $1.0 \mathrm{molL}^{-1}$ sodium hydroxide and $7.8 \times 10^{-6} \mathrm{molL}^{-1}$ of dithizone solution were dissolved in $1.5 \mathrm{~mL}$ of 2-propanol. Then, $0.50 \mathrm{~mL}$ of chloroform was mixed with $10 \mathrm{~mL}$ of the sample solution. The obtained sample was centrifuged for 3 min at 3,000 rpm. Subsequently, $0.40 \mathrm{~mL}$ of the sedimented organic liquid phase was diluted fivefold with chloroform. The absorption spectra of the organic and aqueous phases were measured at $516 \mathrm{~nm}$ using an ultraviolet-visible (UV-Vis) spectrophotometer. 


\section{Results and Discussion}

When a sample solution containing Cd(II) was added to a 2-propanol solution containing a small amount of chloroform, phase separation occurred rapidly, and a small amount of the extraction phase was generated. Figure 1 shows the principle of this phase separation phenomenon. After extracting a sample with a concentration of $3.0 \mu \mathrm{gL}^{-1}$, the measurement results of the concentration of cadmium in the concentrated phase $(0.40 \mathrm{~mL})$ and the aqueous phase $(10 \mathrm{~mL})$ were $0.16 \mathrm{mgL}^{-1}, 0.80 \mu \mathrm{gL}^{-1}$. From these results, the extraction rate of this method was $88 \%$. Chloroform and 2-propanol were rendered homogeneously miscible by solvating chloroform with 2-propanol. However, 2-propanol was diluted by adding a water-based sample to the mixed solution. Therefore, chloroform could not be dissolved, meaning that it eventually sedimented, as 2-propanol lost its function as a solvated molecule by dilution. Similar phase separations have been reported with water/polar organic solvent/non-polar organic solvent systems ${ }^{13-15}$. Figure 2 shows the absorption spectrum of the concentrated Cd (II)-dithizone complex by this method. A spectrum with a maximum absorption wavelength at a wavelength of $516 \mathrm{~nm}$ was observed.

Figure 3 shows the influence of the amount of chloroform used on the volume of the extraction phase. This result shows that the amount of chloroform used can control the volume of the extraction phase. In this study, When the volume of the concentrated phase is reduced, the concentration ratio increases, but the time required for phase separation increases. Therefore, $0.50 \mathrm{~mL}$ of chloroform was set as the optimum condition which has a sufficient concentration ratio and is easy to operate. Under these optimized conditions, an extraction percentage of $88 \%$ was achieved, and the concentration factor was improved 34-fold (from $14 \mathrm{~mL}$ to $0.41 \mathrm{~mL}$ ).

Using the spectrophotometer, the detection limit $(3 \sigma)$ of $1.6 \mathrm{ppb}$ was determined. 
The relative standard deviation $(\mathrm{RSD})$ at $10 \mathrm{ppb}$ was $4.8 \%(\mathrm{n}=5)$. The detection limit of cadmium in the conventional dithizone method is $100 \mu \mathrm{gL}^{-1}$. This result indicate that this method proposed method is a better method than the conventional method. In addition, by using this method, it has become possible to measure the environmental standard value of $3.0 \mu \mathrm{g} \mathrm{\textrm {L } ^ { - 1 }}$.

As dithizone forms colored complexes with most metal ions, it is also used for the colorimetric analysis of heavy metal ions other than $\mathrm{Cd}$. This indicates that the dithizone method lacks selectivity. However, under strongly basic conditions, most metal ions form hydroxide precipitates, meaning that the method's selectivity for $\mathrm{Cd}$ increases ${ }^{16}$. Therefore, in this study, Cd was determined under basic conditions. The influences of coexisting ions was examined. Almost none of the cations and anions, except for $\mathrm{Mg}$ (II) and Ni(II) ions, affected the efficacy of the proposed method. The ions that did affect the proposed method resembled those that are also known to influence the conventional dithizone colorimetric method ${ }^{17}$. However, this influence was reduced by the addition of $\mathrm{Na}_{2} \mathrm{CO}_{3}$ in the case of $\mathrm{Mg}(\mathrm{II})$. Although magnesium ions form a precipitate with carbonate ions, $\mathrm{Cd}$ ions do not, because they exist as anions in a basic state. Therefore, the addition of sodium carbonate appears to have reduced the interference of magnesium ions. To verify the validity of the proposed method for determining $\mathrm{Cd}(\mathrm{II})$, we determined the $\mathrm{Cd}(\mathrm{II})$ concentration in standard river water for a known amount of added $\mathrm{Cd}(\mathrm{II})$. A reasonable recovery rate was obtained for all samples (Table 2), establishing the method's applicability to real environmental samples.

\section{Conclusions}

In this study, the $\mathrm{Cd}(\mathrm{II})$-dithizone complex was extracted into a small amount of the extracted phase using phase separation with water, 2-propanol, and chloroform. 
Using this method, trace levels of Cd(II) can be successfully quantified. Furthermore, this method was employed for the determination of $\mathrm{Cd}(\mathrm{II})$ concentration in standard river water. Although there exists scope for improvement in terms of interference from coexisting substances, the proposed method was successfully adapted for samples with low concentrations of coexisting substances via the use of a masking agent.

\section{Acknowledgements}

This work was financially supported by JSPS KAKENHI Grant Number 20K12153. We would like to thank Editage (www.editage.jp) for English language editing.

\section{References}

1. U.S. Department of Health and Human Services, Public Health Service Agency for Toxic Substances and Disease Registry, "Toxicological Profile for Cadmium”, 2012, 2.

2. K. Aoshima, Tohoku J. Exp. Med., 1987,152, 151.

3. K. Aoshima, Jpn. J. Hyg., 2012, 67, 455.

4. M. Ikeda, T. Watanabe, H. Nakatsuka, J. Moriguchi, S. Sakuragi, F. Ohashi, and S. Shimbo, Food Saf., 2015, 3, 118.

5. World Health Organization, Guidelines for Drinking-water Quality, 4th ed., 2011,180.

6. JIS K 0102, “Water quality-Determination of 33 elements by inductively coupled plasma atomic emission spectroscopy”, 2016, Japanese Industrial Standards Committee, Tokyo.

7. JIS K 0102, “Water quality-Determination of cadmium by atomic absorption spectrometry”, 2016, Japanese Industrial Standards Committee, Tokyo.

8. T. Ashizawa, Jpn. Anal., 1961, 10, 817.

9. H. Akaiwa, H.Kawamoto, and E.Hiyamata, Anal. Sci. 1985, 1, 297.

10.Y. Takagai, T. Maekoya, and S. Igarashi, J. Chem. Soc. Jpn., 2000, 4, 291.

11.S. Oshite, M. Furukawa, and S. Igarashi, Analyst, 2001, 126, 703. 
12. S. Oshite, S. Igarashi, Anal. Sci., 2001, 17 (Supplement):i1329.

13. T. Kato, Y. Nagashima, A. Manaka, C. Nakamura, S. Oshite, and S. Igarashi, Anal. Sci, 2019, 35, 939.

14. Y. Yokota, A. Manaka, M.Tafu, T. Kato, K. Tomita, and T. Akazawa, Bull. Chem. Soc. Jpn., 2019, 92, 807.

15.S. Igarashi, T. Arai, and T. Kawakami, Bunseki Kagaku, 1994, 43, 1183.

16. T. Ashizaw, Jpn. Anal., 1970, 19, 1333.

17. M. Moritsugu, Jpn. Anal., 1964, 13, 64. 
Table 1. Analytical results for $\mathrm{Cd}(\mathrm{II})$ in standard river water.

$$
\left[\mathrm{Cd}^{2+}\right] / \mathrm{mgL}^{-1} \quad \text { Recovery, R.S.D., }
$$

Sample

$\begin{array}{llll}\text { Content } & \text { Added } & \text { Found } & \%\end{array}$

$\begin{array}{llllll}\text { No.1 } & 0.0010 & 0.010 & 0.011 & 98 & 2.4\end{array}$

$\begin{array}{llllll}\text { No.2 } & 0.0010 & 0.020 & 0.021 & 100 & 0.50\end{array}$




\section{Figure Captions}

Fig. 1 principle of proposed phase separation phenomenon.

[dithizone $]=2.0 \mathrm{ppm},[\mathrm{NaOH}]=0.10 \mathrm{~mol} \mathrm{~L}^{-1}, 2$-propanol:1.5 mL, chloroform: $0.50 \mathrm{~mL}$

Fig.2 UV-vis spectrum of Cd(II)-dithizone

$\left[\mathrm{Cd}^{2+}\right]=0.020 \mathrm{mg} \mathrm{L}^{-1}$

Fig. 3 Influence of the amount of reagent used on the volume of the extraction phase.

$\mathrm{CHCl}_{3}:$ 2-propanol $=1: 3,\left[\mathrm{Cd}^{2+}\right]=0.010 \mathrm{mg} \mathrm{L}^{-1},[\mathrm{NaOH}]=1.0 \mathrm{~mol} \mathrm{~L}^{-1}$,

[dithizone $]=2.0 \mathrm{ppm}$

--- : optimum condition(Addition:500 $\mu \mathrm{L}$, extraction phase:410 $\mu \mathrm{L}$ ) 

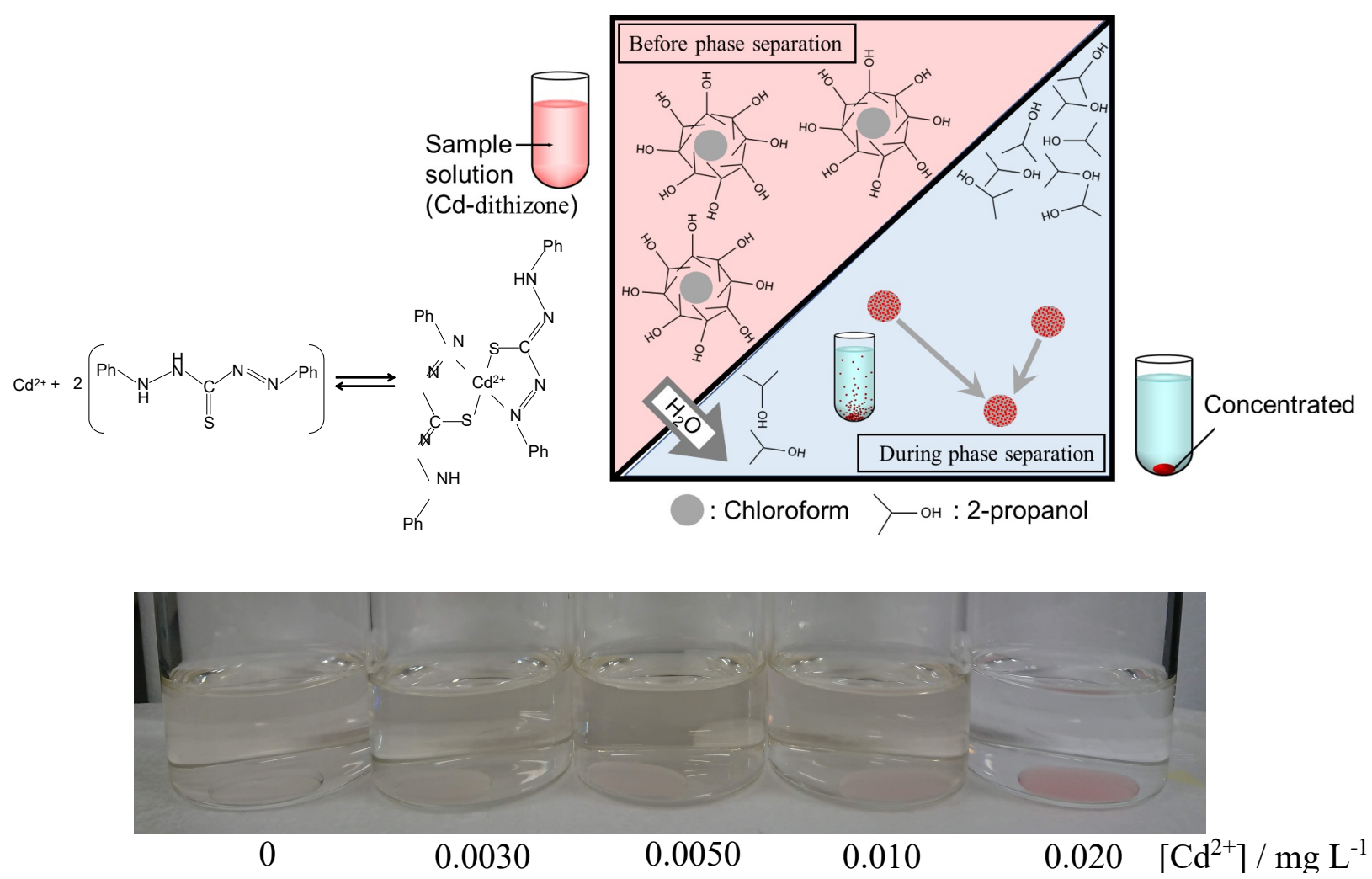

Fig.1 


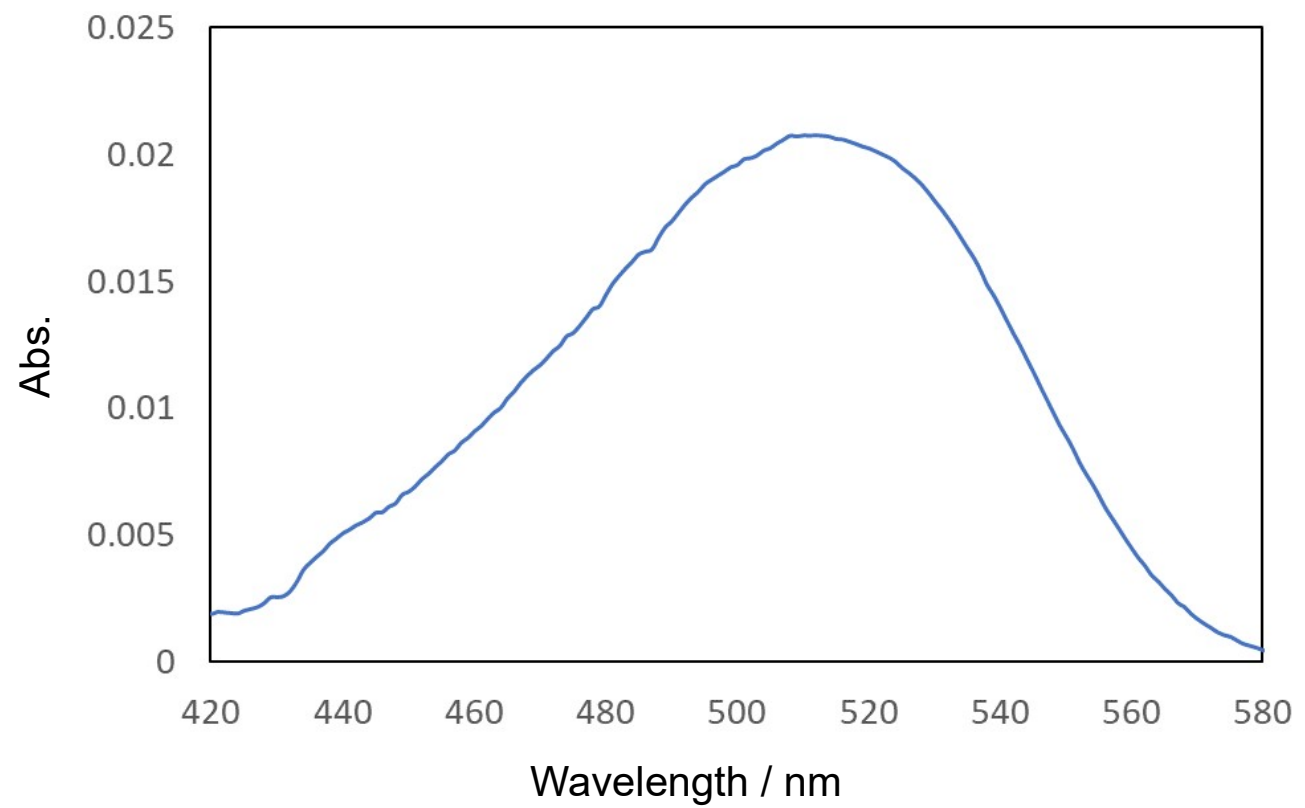

Fig.2 


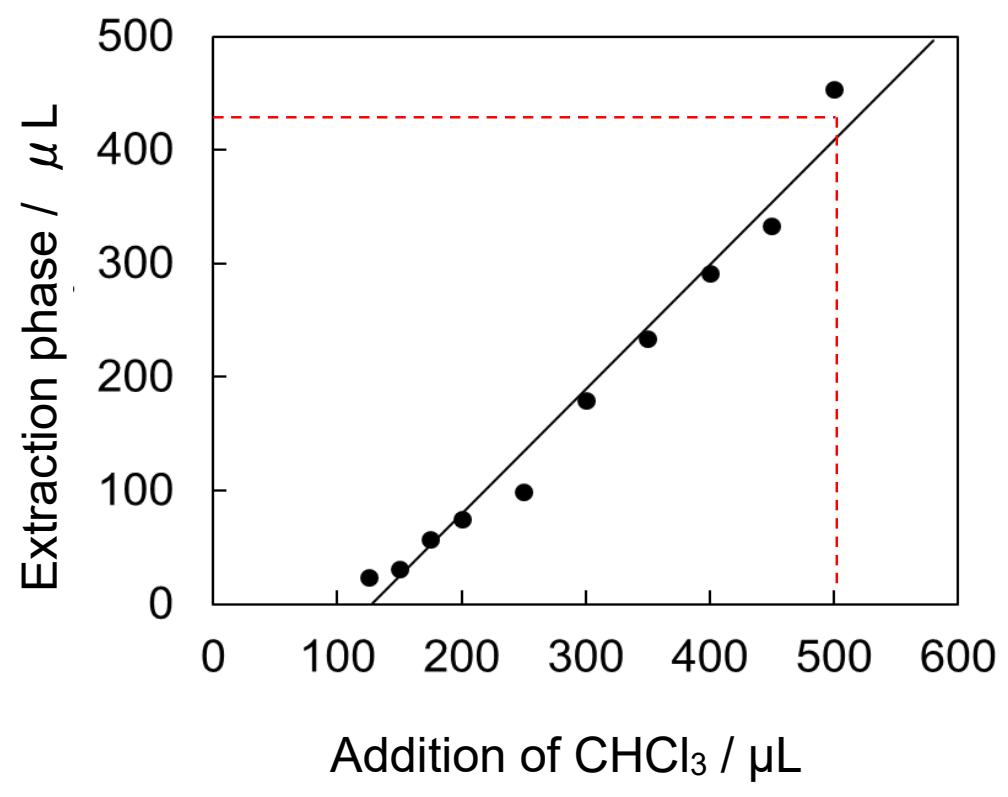

Fig. 3 


\section{Graphical Index}

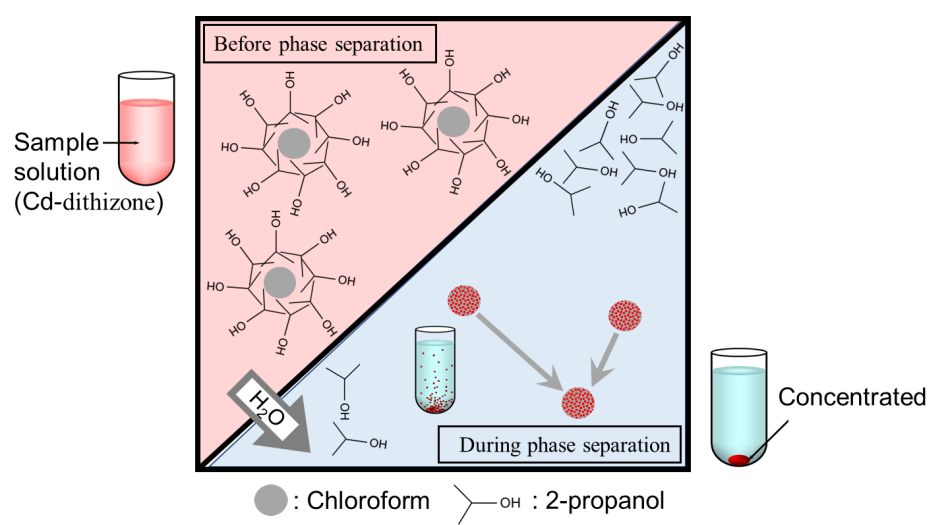

